Editorial

\title{
Pulse Synchronized Contractions (PSCs)
}

\section{Katherine A Lothman* and Allen W Mangel}

RTI Health Solutions, Research Triangle Park, NC, USA

A key platform underpinning the traditional understanding of the cardiovascular system, with respect to the behavior of large arterial vessels, is Otto Frank's Windkessel Hypothesis [1]. This hypothesis posits simply that the smooth muscle walls of large arteries do not undergo rhythmic contractions in synchrony with the heartbeat but, rather, behave as passive elastic tubes undergoing distension from pulsatile pressure waves. The Windkessel Hypothesis is elegant, well described for over a century, ingrained in the understanding of cardiovascular medicine and physiology, and simply wrong.

Several groups have now shown that the arterial smooth muscle wall undergoes rhythmic activation in synchrony with the heartbeat in a variety of tissues, including human brachial artery; canine coronary, femoral, and carotid arteries; rabbit aorta; feline pulmonary artery and rodent aorta [2-8]. The phasing of these events is such that the upstroke of the contraction slightly precedes the upstroke of the pulse wave, suggesting nomenclature for the events as pulse synchronized contractions, or PSCs [3,6-8].

PSCs have been found to be of neurogenic origin, sensitive to the neural blocker tetrodotoxin $[3,8]$. Although the specific neural pathways regulating PSCs have not been elucidated, the alpha-adrenergic system is at least partially involved, as evidenced by reduction or blockade of PSCs by the alphaadrenergic blocker phentolamine [8]. Further, PSCs have not been observed following vessel excision in in vitro studies, as an intact nervous system is not present. The pacemaker for the PSC resides in the right atrium, as suggested by two lines of evidence. First, pacing of the right atrial region to faster than spontaneous frequencies leads to a one-to-one correspondence of PSC frequency with the stimulation rate [3]. Additionally, excision of the right, but not the left, atrial appendage results in elimination of PSCs [3]. As the pacemaker region for PSCs and the heartbeat both lie in the right atrium, this may potentially allow for coordination between the heartbeat and pulse wave with PSCs $[3,5,8]$. Extensive evaluations also have been performed showing the PSC was not an artifact produced either by cardiac contractility or from the vessel distension from the pulse wave $[3,5,6]$.

\section{More Information}

*Address for Correspondence: Katherine A Lothman, RTI Health Solutions, 3040 East Cornwallis Road, PO Box 12194, Research Triangle Park, NC 27709-2194, USA, Tel: +1.919.541.6216; Email: klothman@rti.org

\section{Submitted: 24 October 2019 \\ Approved: 15 November 2019 Published: 15 November 2019}

How to cite this article: Lothman KA, Mangel AW. Pulse Synchronized Contractions (PSCs). J Cardiol Cardiovasc Med. 2019; 4: 199-200.

DOI: dx.doi.org/10.29328/journal.jccm.1001067

Copyright: @ 2019 Lothman KA, et al. This is an open access article distributed under the Creative Commons Attribution License, which permits unrestricted use, distribution, and reproduction in any medium, provided the original work is properly cited.

Check for updates

Prokop, et al. [9] have shown that one of the most important causative factors leading to intimal dissection is the rate of rise of the pulse wave $(\mathrm{dP} / \mathrm{dT})$. Previously, we hypothesized that PSCs may serve a vessel protective function such that contraction of the vessel from the PSC, at the time of maximum distension from the pulse wave, can serve to reduce the Laplacian Forces acting on the wall $[3,5,7]$. Recognition of the PSC suggests several potential lines of investigation that should be pursued.

What are the consequences of in vivo disruption of PSCs on the hemodynamic parameters in chronic animal models, and what are the anatomic changes to the vessel wall after disruption of PSCs?

What specific neural pathways (e.g., local ganglia versus central autonomic neurons) are involved in PSC generation?

Are attempts to develop noninvasive techniques for observing PSCs (e.g., ultrasonic) and noting abnormal activity in humans with aortic aneurysms and other clinical findings warranted?

\section{References}

1. Frank O. Die Grundform des Arteriellen Pulses. Zeitschrift fur Biologie 37: 483-526. (1899). J Mol Cell Cardiol. 1990; 22: 253-254.

PubMed: https://www.ncbi.nlm.nih.gov/pubmed/2192068

2. Heyman F. Movements of the arterial wall connected with auricular systole seen in cases of atrioventricular heart block. Acta Medica Scandinavica. 1955; 152: 91-96.

PubMed: https://www.ncbi.nlm.nih.gov/pubmed/13258159

3. Mangel A, Fahim M, van Breemen C. Control of vascular contractility 
by the cardiac pacemaker. Science. 1982; 215: 1627-1629. PubMed: https://www.ncbi.nlm.nih.gov/pubmed/7071582

4. Ravi K, Fahim M. Rhythmic contractile activity of the pulmonary artery studied in vivo in cats. J Autonomic Nervous System. 1987; 18: 33-37. PubMed: https://www.ncbi.nlm.nih.gov/pubmed/3819314

5. Mangel A. Does the aortic smooth muscle wall undergo rhythmic contractions during the cardiac cycle? Experimental and Clinical Cardiology. 2014; 20: 6844-6851.

6. Mangel A, Lothman K. Emergence of a new paradigm in understanding the cardiovascular system: pulse synchronized contractions. Cardiovasc Pharm. 2017; 6: 5.
7. Mangel A, Lothman K. Pulse synchronized contractions (PSCs): a call to action. Open J Cardiol Heart Dis. 2018; 1: OJCHD.000508.

8. Sahibzada N, Mangel AW, Tatge JE, Dretchen KL, Franz MR, et al. Rhythmic aortic contractions induced by electrical stimulation in vivo in the rat. PLoS One. 2015; 10: e0130255.

PubMed: https://www.ncbi.nlm.nih.gov/pubmed/26132172

9. Prokop EK, Palmer RF, Weat MW Jr. Hydrodynamic forces in dissecting aneurysms: in-vitro studies in a Tygon model and in dog aorta. Circ Res. 1970; 27: 121-127.

PubMed: https://www.ncbi.nlm.nih.gov/pubmed/5424561 